\title{
Implementation of Automatic Aircraft Tracking with RTL-SDR
}

\author{
Dr. Yedukondalu Kamatham ${ }^{1}$ and Sushmitha Pollamoni ${ }^{2}$ \\ ${ }^{1}$ Professor, CVR College of Engineering/ECE Department, Hyderabad, India \\ Email: kyedukondalu@gamil.com \\ ${ }^{2}$ PG Scholar, CVR College of Engineering/ECE Department, Hyderabad, India \\ Email: spullamoni@gmail.com
}

\begin{abstract}
To improve the efficiency, safety, and capacity of air space system, an Automatic Dependent SurveillanceBroadcast (ADS-B) is one of the popular technologies used in air traffic surveillance. The ADS-B uses $1090 \mathrm{MHz}$ band. ADS$B$ is complemented by existing radar-based technologies to locate aircraft. The coexistence of radar systems and ADS-B is a key system to detect and resolve conflicts in the Next Generation Air Transportation System (NGATS). But, the major disadvantage of ADS-B is its implementation complexity and increase in cost without user-friendly. This paper focuses to reduce the complexity and cost of ADS-B system implementation in MATLAB with the help of Software Defined Radio (SDR). SDR is user-friendly and easy to handle aircraft information without increasing the cost. The ADS-B system implemented with SDR can receive the multiple numbers of aircraft information such as altitude, latitude, longitude, speed, and direction in real-time. Usage of SDR maximizes the information coverage with reliability and can achieve timely communication in Air Traffic Control (ATC) networks.
\end{abstract}

Index Terms: SDR, ADS-B, ATC, NGATS

\section{I.INTRODUCTION}

Nowadays, with the increase in demand of National Air Space Systems (NAS), Federal Aviation Administration (FAA) faces number of challenges due to fundamental limitations of current radar systems. The air transportation systems are modernized by research and development programs of growth in air traffic levels of NAS. The main objective of an air transportation system is to provide safety and efficiency. Airspace is divided into two sectors in which each sector has Air Traffic Controller (ATC) responsible for managing air traffic. Sector in an aircraft is centrally controlled by corresponding ATC [1]. In U.S., Next Generation Air Transportation System (NGATS) is referred as a future system.

In airspace, the most congested region of air traffic (information flow) is controlled by centralization results in most efficient operations. Nevertheless, the implementation of such centralization on large scale is most expensive which requires all aircraft information to be relayed to the central facility through surveillance. This problem is aggravated as traffic demand increases. Similarly, in low traffic density, the aircraft interactions are less, and centralization efficiency is more as a distributed control strategy (aircraft unilaterally resolves in skirmish). The multiple aircraft in distributed system, that cooperate for the safety and efficiency of air traffic management.

To provide centralized surveillance, current systems depend on ground-based radar systems. Deployment of ground radar systems is very expensive and complex to maintain [2]. In areas, the system without line of sight, cannot provide coverage and subjected to terrain blockage. Instead of using expensive ground radar systems, NGATS aircraft will have wireless communication platform with enhanced onboard sensing capabilities [3]. The system in an area with line of sight constraints, the wireless systems can operate and enable cooperative techniques.

The primary target of FAA's NGATS plan is to transform the air transportation system into an adaptive, flexible and highly automated system capable of handling the expected increase in air traffic. The Cyber-Physical Systems (CPS) primary example is NGATS in which computations for monitoring, optimization, and sensing are tightly coupled with the actions of the aircraft themselves [4].

According to the NGATS plan, the design of Automatic Dependent Surveillance-Broadcast (ADS-B) is to increase the capacity, safety, and efficiency of NAS by sharing enhanced information between aircraft and Air Traffic Control (ATC) facilities [5]. ADS-B frequently transmits aircraft information such as aircraft number, position, altitude, speed and directions are determined by using satellite-based navigation systems. ADS-B shares frequency band $1090 \mathrm{MHz}$, which is same as current radar systems. ADS-B is less expensive when compared to conventional radar systems [6].

This paper deals with a low-cost solution for automatic aircraft tracking with Software Defined Radio (SDR). The SDR is used for ADS-B implementation of a real-time air traffic monitoring system. SDR is used to provide rapidly prototyping high-performance wireless communication systems with integrated software solutions [7]. SDR is a Universal Serial Bus (USB) device with low cost which receives RF radio signals. This device is designed for DVB$\mathrm{T}$ (Digital Video Broadcast-Terrestrial) receivers. By handling SDR in different modes, to receive any kind of signal in the range of frequency tuner operates. This device is designed to receive not only Digital Television (DTV) signals but also receive all radio frequency signals in specified frequency range [8], [9]. The range of SDR is different from device to device depending on the components mostly used. However, SDR operates in the frequency range of $25 \mathrm{MHz}$ to $1.75 \mathrm{GHz}$. The RTL-SDR front-end receives RF signals live off the air, down converted to form baseband signal, digitizes them and the output is the samples of baseband signal [10]. 
The rest of this paper is organized as follows: Section II deals with functionality of ADS-B and Section III presents implementation of ADS-B with RTL-SDR. Section IV represents results and discussions and the conclusion of this paper is presented in section $\mathrm{V}$.

\section{ADS-B WITH ATC}

The air traffic controller (ATC) provides advisory service to aircraft from ground in non-controlled airspace. The main aim of ATC is to prevent collisions, expedite the flow of air traffic. ATC provides security to defensive role, operated by military in some countries. In many countries, depending on the operating range of airspace, the services are provided to all private, military aircraft, which depends upon the class of airspace and type of aircraft, ATC releases instructions that pilot should obey.

ADS-B is defined as an Automatic Dependent Surveillance-Broadcast which periodically broadcasts signals for tracking aircraft to determine its position by satellite navigation. The ATC on ground stations receives the information and ADS-B is a second surveillance radar. By providing the situational awareness of air traffic, the information is received by other aircraft which lead to selfseparation. ADS-B is 'automatic' and does not require external input or pilot. It is dependent on aircraft navigation system data.

ADS-B consists of two services, they are "ADS-B Out and ADS-B In" for controlling the aircraft worldwide as replacing the radar with the primary surveillance method. For upgrading and enhancing aviation infrastructure and operation of NGATS national airspace ADS-B is used. ADS-B is an integral part of NGATS national airspace. With Traffic Information Services-Broadcast (TIS-B) and Flight Information Services-Broadcast (FIS-B) applications, the ADS-B system provides weather information in United States. To provide the information to ATC about the aircraft's visibility and enhances safety in real-time, ADS-B transmits the position and velocity data every second. For inexpensive aircraft tracking, planning and dispatch of data infrastructure are provided by ADS-B. Air Traffic Control is used as primary surveillance radar and secondary surveillance radar is used to know the location and identify the aircraft. Mode S transponder is an aircraft's equipment that is used to provide information to ATC about aircraft. Air Traffic Control Radar Beacon System (ATCRBS) is a secondary radar system currently used by aircraft, to achieve the compatibility between ATCRBS and Mode-S.
The avionics is an electronic system, which is used in aircraft, artificial satellites, and spacecraft. This system includes communication, navigation, display, management of multiple systems and hundreds of systems are fitted to aircraft to perform individual functions. This can be as simple as searchlight for police helicopter. By mode-S transponder, ADS-B, multichannel Distance Measuring Equipment (DME) minimizes size of avionics used in Aeronautical Radio Navigation Services (ARNS).

\section{IMPLEMENTATION OF ADS-B WITH RTL-SDR}

ADS-B receives signals with the help of Mode-S signal scheme. Mode- $\mathrm{S}$ is an aviation transponder interrogation mode type. When the interrogation request is received by an aircraft, the request is sent back to squawk code of transponder. Mode-S is designed with following properties as given in Table 1. ADS-B messages contain information about Altitude, Aircraft ID (24-bit Sequence).

TABLE I.

INPUT PARAMETERS

\begin{tabular}{|l|l|}
\hline Transmit frequency & $1090 \mathrm{MHz}$ \\
\hline Modulation & Pulse Code Modulation \\
\hline Data rate & $1 \mathrm{Mbps}$ \\
\hline Short Squitter Length & $59 \mu \mathrm{sec}$ \\
\hline Extended Squitter Length & $112 \mu \mathrm{sec}$ \\
\hline
\end{tabular}

In Figure 1, at a physical layer, RTL-SDR is designed and it acts as a source. SDR samples the received signal at a rate of $2.4 \mathrm{MHz}$ with interpolation factor of 5 to a practical sampling rate of $12 \mathrm{MHz}$ with data rate of $1 \mathrm{Mbps}$. The packet synchronizer and Mode-S transponder are in a physical layer, work on sub-frames of data equivalent to squitter packets. The synchronizer first correlates the received signal with 8 - $\mu$ sec preamble and then a peak value is determined. The validation synchronization point is obtained by checking the preamble sequence either matches or not. The Mode-S data format is shown in Table II. The received messages of shown on a Graphical User Interface (GUI) by Data viewer. According to received messages, the time gets updated. if received signal time $(t)$ is less than delay time $\left(t_{\text {gnd }}\right)$ then signal gets processed to the physical layer and displays the experimental view of ADS-B as shown in Figure 2. With the help of NooElec software, the SDR drivers are installed in MATLAB. The SDR with range of $75 \mathrm{MHz}$ to $1.25 \mathrm{GHz}$ is used in this work for tracking the information of aircraft. Figure 3 shows the NooElec RTLSDR, procured at a price of Rs 4000 is used for this experimental work.

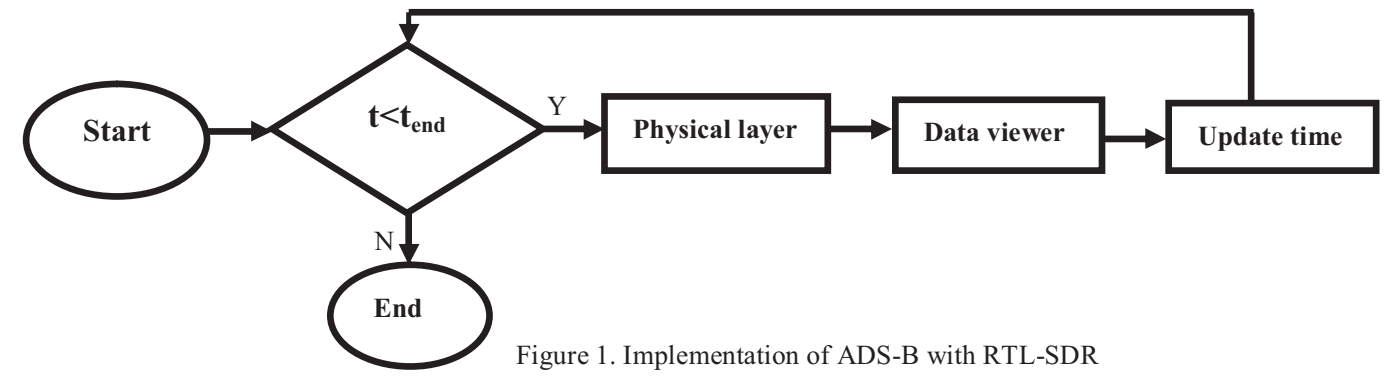

CVR College of Engineering 


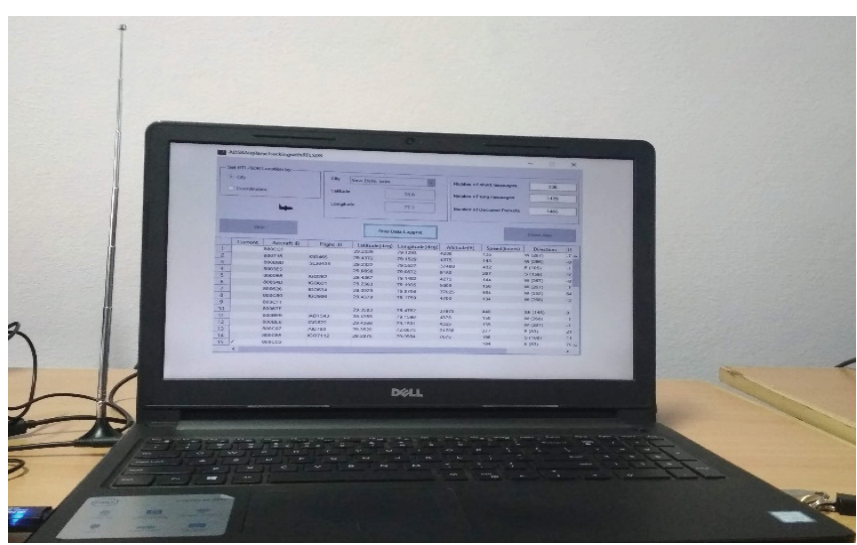

Figure 2. Experimental Setup of ADS-B

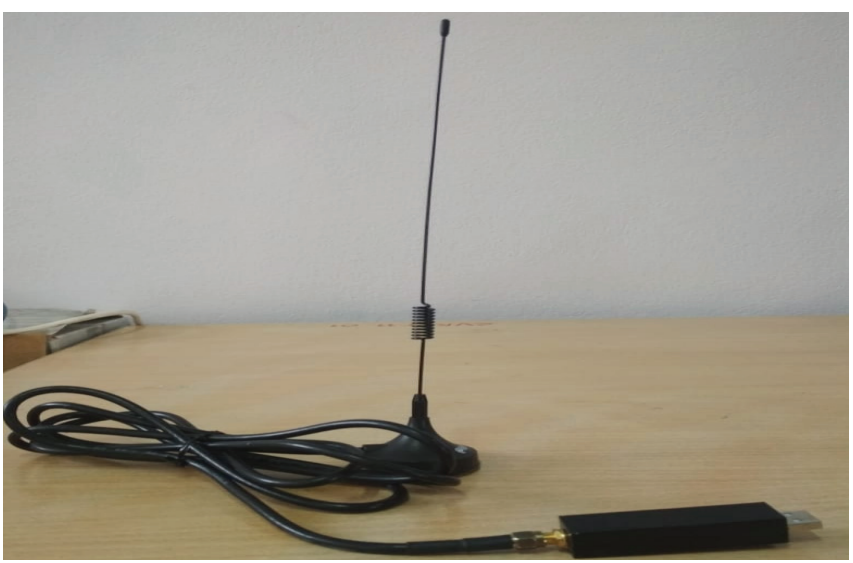

Figure 3. RTL-SDR diagram

TABLE II.

SQUITTER LENGTH INFORMATION

\begin{tabular}{|c|c|l|l|}
\hline S.No. & Bit size & Bits & Name \\
\hline 1 & 5 & $1-5$ & Downlink Format \\
\hline 2 & 3 & $6-8$ & Capability \\
\hline 3 & 24 & $9-32$ & ICAO Aircraft Address \\
\hline 4 & 5 & $33-37$ & Type Code \\
\hline 5 & 51 & $38-88$ & Data \\
\hline 6 & 24 & $89-112$ & Parity ID \\
\hline
\end{tabular}

Mode-S signal format with sync pulse i.e. $8 \mu$ sec long followed for both 56 and $112 \mu \mathrm{sec}$. The data representation is shown in Figure 4.

\section{A. Decoding Technique}

The decoding process of ADS-B is shown in Table 2 has 5 parts with 112 bits long: Downlink format (DF) (5 bits) Capability (CA) (3 bits) - ICAO (24 bits) - Data (56 bits) Parity Identity (PI) (24 bits).

\section{B. ADS-B Message types}

The basic relationship between each type of code and information contained in the DATA segment is given in Table 3. For calculating Latitude, Longitude and Altitude values of an aircraft following expressions are used with Compact Position Reporting (CPR).
TABLE III.

INFORMATION ABOUT CODES

\begin{tabular}{|c|c|l|}
\hline S.No. & TC & Content \\
\hline 1 & $1-4$ & Aircraft Identification \\
\hline 2 & $5-8$ & Surface Position \\
\hline 3 & $9-18$ & Airborne Position (w/Baro Altitude) \\
\hline 3 & 19 & Airborne Velocities \\
\hline 4 & $20-22$ & Airborne Positions (w/GNSS Height) \\
\hline 5 & $23-31$ & Reserved for other uses \\
\hline
\end{tabular}

\section{Calculation of Latitude}

$$
j=\text { floor }\left(59+\text { Lat }_{\text {cpreven }}-60+\text { Lat }_{\text {cpreven }}+\frac{1}{2}\right)
$$

First, two constants will be used

$$
\begin{aligned}
& \text { L Lat }_{\text {even }}=\frac{360}{4 * N Z} \\
& d \text { Lat }_{\text {odd }}=\frac{360}{4 * N Z-1}
\end{aligned}
$$

Where NZ is a present latitude value. For computing latitude values the following expressions are considered:

$$
\begin{aligned}
& \text { Lat }_{\text {even }}=d L a t_{\text {even }} *\left(\bmod (j .60)+\text { Lat }_{\text {cpr Even }}\right. \\
& L a t_{\text {odd }}=d L a t_{\text {odd }} *\left(\bmod (j .59)+L_{\text {aprodd }}\right.
\end{aligned}
$$

Final latitude is chosen depending on the timestamp of the frames:

$$
\text { Lat }_{\text {odd }}=\left\{\begin{array}{c}
\text { Lat }_{\text {even }} \text { if }\left(T_{\text {even }} \geq T_{\text {odd }}\right) \\
\text { Lat }_{\text {odd }}
\end{array}\right.
$$

\section{Calculation of Longitude}

For an Even frame:

$$
\begin{aligned}
& n i=\max \left(N L\left(\text { Lat }_{\text {even }}\right), 1\right) \\
& d L o n=\frac{360}{n i}
\end{aligned}
$$

$$
\begin{aligned}
& m=\text { floor }\left(\begin{array}{c}
\text { Lon }_{\text {epreven }} *\left[N L\left(\text { Lat }_{\text {even }}\right)-1\right]- \\
\text { Lon }_{\text {epr odd }} * N L\left(\text { Lat }_{\text {odd }}\right)+\frac{1}{2}
\end{array}\right) \\
& \text { Lon }=d l o n *\left(\bmod (m, n i)+\text { Lon }_{\text {cpreven }}\right)
\end{aligned}
$$

For an Odd frame: $n i=\max \left(N L\left(L_{a t_{\text {Odd }}}\right)-1,1\right)$

$$
d L o n=\frac{360}{n i}
$$



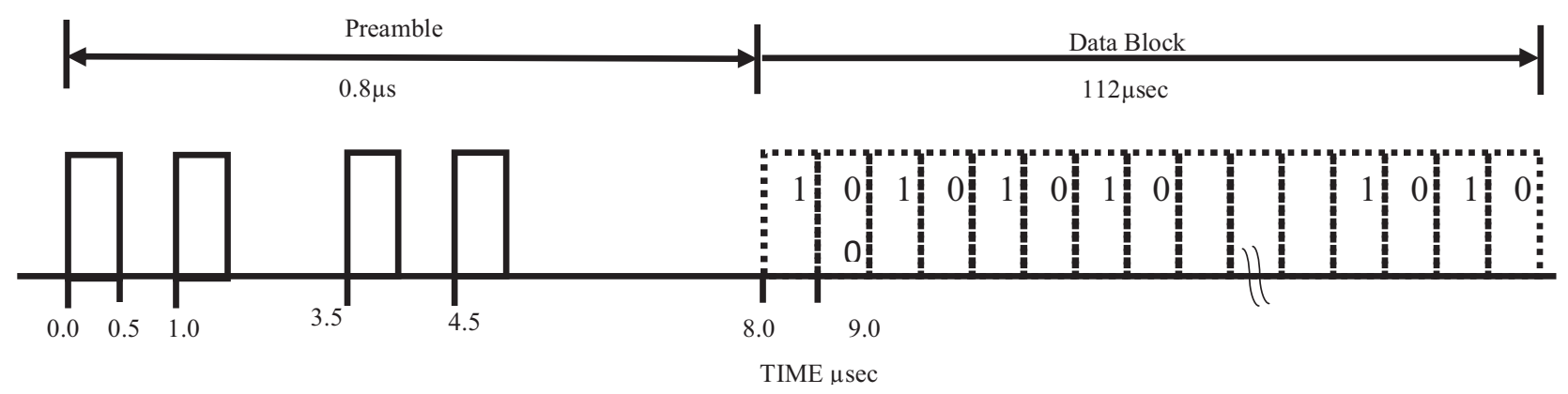

Figure 4. Mode-S data representation

$$
m=\text { floor }\left(\begin{array}{c}
\operatorname{Lon}_{\text {epreven }} *\left[N L\left(\text { Lat }_{\text {even }}\right)-1\right]- \\
\operatorname{Lon}_{\text {epr odd }} * N L\left(\text { Lat }_{\text {odd }}\right)+\frac{1}{2}
\end{array}\right)
$$

$$
\text { Lon }=d l o n *\left(\bmod (m, n i)+\text { Lon }_{\text {cpreven }}\right)
$$

\section{E. Calculation of Altitude}

From the data frame, the altitude of aircraft is calculated. This Q-bit (bit 48) indicates whether the altitude is encoded in multiples of 25 or $100 \mathrm{ft}$ and NQ denotes the N number of times of $\mathrm{Q}$ bits in multiple signals. The final altitude value will be:

$$
\text { Alt }=N Q 25-1000(f t)
$$

Finally, for tracking of aircraft Latitude, Longitude, and Altitude are obtained.

\section{RESUlts AND Discussions}

The RTL SDR receives the radio frequency signals of ADS-B. To display the information, the ADS-B Airplane Tracking Window is displayed. To track the exact location with SDR, depending on the requirements the city or coordinates are selected. If city is selected, then latitude and longitude values will be displayed automatically i.e. for example: in Figure 5, New Delhi is selected as the city; latitude and longitude values are displayed as Latitude 28.5 and Longitude - 77.2. The SDR starts receiving the tracking the Aircraft information. From the aircraft the short messages and long messages are received and displayed. Here short messages are displayed as 336 and long messages are displayed as 1129. The received short and long messages are decoded and displayed as 1465.

D $A$

ADSBAirplane TrackingwithRILSDR

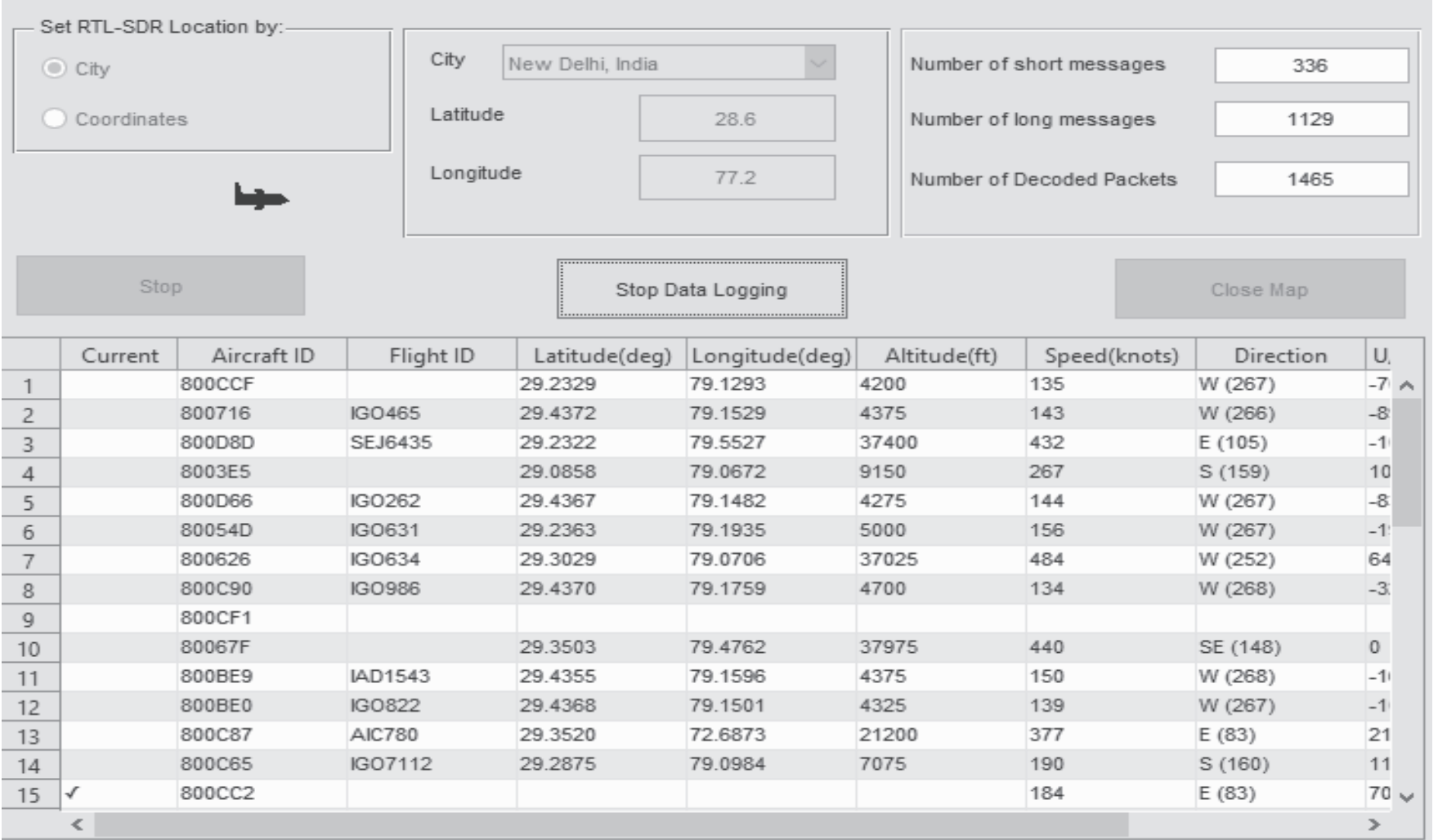

Figure 5. Aircraft tracking information 


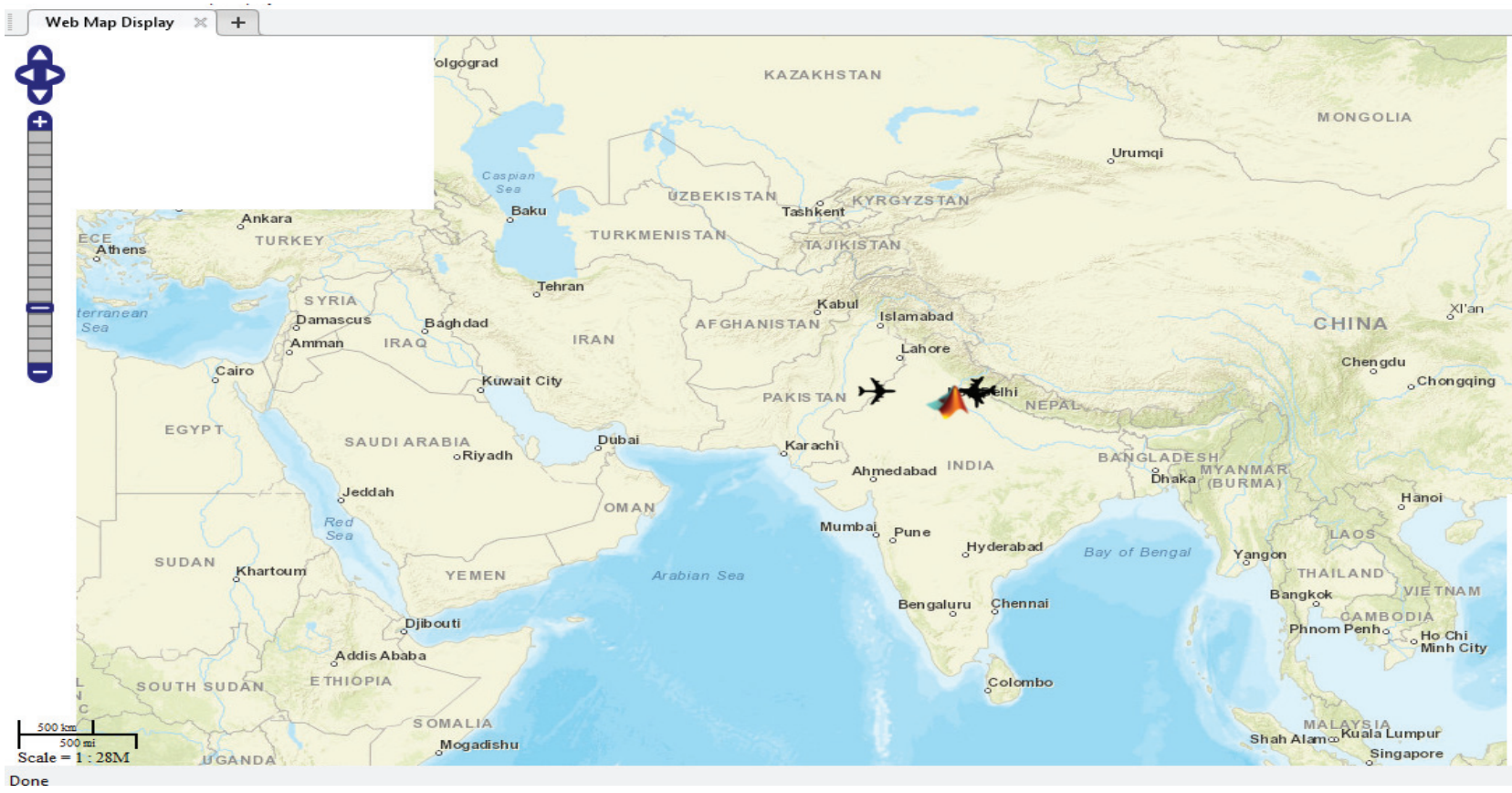

Figure 6. Geographical map of Airplanes tracking

With the selection of city of the specified latitude and longitude, the aircraft are tracked and displayed with Aircraft ID, Flight ID, Latitude (deg), Longitude (deg), Altitude (ft), Speed (knots), Direction. The current aircraft information is displayed with $\sqrt{ }$ (tick) mark. For example, current aircraft tracking is displayed with the Aircraft ID800716, Aircraft ID-IGO465, Latitude-29.4372, Longitude79.1529, Altitude-4375 feet, Speed-143 knots, DirectionWest $\left(266^{\circ}\right)$.

The aircraft is displayed in the world map shown in Figure 6 according to data tracked like time, altitude, direction. To display all aircrafts in this map to and fro to New Delhi, this city latitude and longitude values should be selected from the Figure 5. The background of map can also change according to requirement of either local map or geographical map. If we point cursor on aircraft image in Figure. 6, it displays the timing of aircraft and altitude, latitude and longitude of aircraft. If coordinates selected, according to requirements latitude and longitude values should be entered, and same process is repeated for this procedure. If this RTL-SDR is used for tracking of an aircraft, bulky equipment (used in conventional tracking systems) is not required so that complexity, cost of system is drastically reduced.

\section{CONCLUSIONS}

In this paper, ATC becomes the most prominent technology in surveillance system. ADS-B is also most important part of ATC for aircraft tracking systems in ATC because it provides aircraft information. To reduce the cost and complexity of ADS-B system, the system is implemented with help of RTL-SDR. The RTL-SDR is user friendly and easy for tracking. SDR receives the aircraft information with altitude, longitude, latitude and tracked aircraft are displayed. According to the aircraft gets tracked, the information is shown in map. The ADS-B system is used in many surveillance applications for tracking aircraft.

\section{REFERENCES}

[1] Next generation implementation plan, Flight Attendant, 2011.

[2] Edward A Lester and R. John. Hansman, "Benefits and Incentives for ADS-B Equipage in the National Airspace System", Mass Institute of Technology, ICAT-2007.

[3] RTCA (firm), SC-186, "Minimum Aviation Performance Standards for Automatic Dependent Surveillance Broadcast (ADS-B)", Washington, DC: RTCA, C1998.

[4] Krishna Sampigethaya, Radha Poovendran, Sudhakar Shetty, Terry Davis and Chuck Roy, "Future E-Enabled Aircraft Communications and Security: The Next 20 Years and Beyond", Proceeding of IEEE, Volume: 99, Issue: 11, pp: 2040-2055, November 2011

[5] RTCA DO-242, "Minimum Aviation System Performance Standard for Automatic Dependent Surveillance Broadcast (ADS-B)", December 2006.

[6] Magaly Varga, Zsolt Alfred Polgar, Horia Hedesiu, "ADS-B Based Real-Time Air Traffic Monitoring System”, $201538^{\text {th }}$ International Conference on Telecommunications and Signal Processing (TSP), pp: 215-219, 2015.

[7] Akshay N, Shruthi R, Sushmitha K N, Vanitha R and Dr. Rekha K R, "Live Aircraft Detection with Mode-S Transponder Using RTL-SDR", International Journal of Advanced Research in Computer Science and Software Engineering, Volume: 7, Issue: 5, pp: 490-496, May 2017.

[8] D. Poirier-Quinot, P. Duvaut, L.Girardeau, B.F.G. Katz, “3D Head-Mounted Antenna Array Architecture Optimization based on the Fisher Information Matrix", IV International Congress on Ultra-Modern Telecommunication and Control Systems 2012, pp:135-142, 2012.

[9] J. Mitola, "The Software Radio Architecture", IEEE Communication Magazine, Volume: 33, Issue: 5, pp: 26-38, May 1995.

[10] G. Gilbert, "Historical Development of the Air Traffic Control System", IEEE Transaction on Communications, Volume: 21, Issue: 5, pp: 364-375, May 1973. 